\title{
An eight-channel computer-controlled scanning electromyograph
}

\author{
ALAN J. FRIDLUND and STEPHEN C. FOWLER \\ University of Mississippi, University, Mississippi 38677
}

\begin{abstract}
A measurement system is described for the computerized detection, digitizing, and storage of electromyographic (EMG) signals from up to eight muscle groups. The use of a channel scanning technique allows almost simultaneous multichannel recording with minimal circuit redundancy. Specifically, the scanning electromyograph allows several EMG preamplifiers to share expensive isolation, signal-conditioning, and interface circuitry. The design incorporates monolithic integrated circuit preamplifiers and includes optical isolation of the preamplifier circuitry for protection of the subject. Hybrid active filter modules allow convenient selection of system bandpass characteristics. Key measurement parameters are software programmable. The scanning electromyograph is inexpensively constructed from readily available componentry. Specifications for all functional blocks within the instrument are comparable to those of commercial instrumentation.
\end{abstract}

In electromyographic research, it is often desirable to monitor several muscle groups concurrently. Studies of posture (Floyd \& Silver, 1955) and gait (Milner, Basmajian, \& Quanbury, 1971), investigations of somatic and facial tensional patterning (Balshan, 1962; Schwartz, Fair, Salt, Mandel, \& Klerman, 1976), and newer multiple-muscle biofeedback procedures (DeBacher \& Basmajian, 1977) may require multichannel recording capability.

Commercial instrumentation typically is quite expensive, due to the precision and sensitivity requisite for the detection of low-level bioelectric phenomena, and to the extensive signal conditioning necessary to convert these phenomena into useful electrical signals.

The concept of the scanning electromyograph (SEMG) allows multichannel recording capability with minimal circuit duplication. Specifically, the SEMG allows several EMG preamplifiers to share signalconditioning and interface circuitry, resulting in circuit economy and little sacrifice in function.

Block diagrams for a standard EMG instrument and the eight-channel SEMG, described in detail herein, are shown in Figure 1. The standard EMG detects myopotentials, or muscle action potentials (MAP), with a high-gain preamplifier, isolates the line-powered circuitry from the user, filters the MAP to maximize signal-to-noise ratio and exclude $60-\mathrm{Hz}$ line interference, rectifies, and then integrates these signals over time for

The authors wish to thank the following for their assistance in implementing this research: K. O. McGraw, B. G. Tate, C. R. Fridlund, A. W. Price, W. P. Copple, and J. H. Bruening. This research was supported in part by the Committee on Faculty Research of the University of Mississippi. Requests for reprints should be addressed to Alan J. Fridlund, P.O. Box 7457, Department of Psychology, University of Mississippi, University, Mississippi 38677. presentation to a display or recording device. ${ }^{1}$ The SEMG acts similarly, except that multiple preamplifiers are scanned and their signals are selectively connected to the isolation, filtering, rectification, integration, and display/recording sections.

The scanning procedure does not afford true simultaneous multichannel recording. Rather, a sample of the electrical activity within each muscle group is taken as the selected sequence of muscle groups is scanned. A tensional profile for each complete scan may thus be assembled. This approach is entirely suitable for studies involving tensional and postural patterning. However, researchers investigating EMG correlates of rapid movement may find continuous multichannel recording preferable but more expensive.

Due to the amount of data involved in multichannel recording and the precision with which they must be

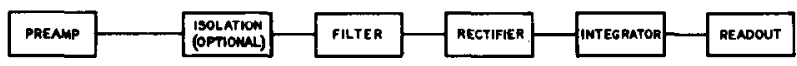

A. STANDARD EMG

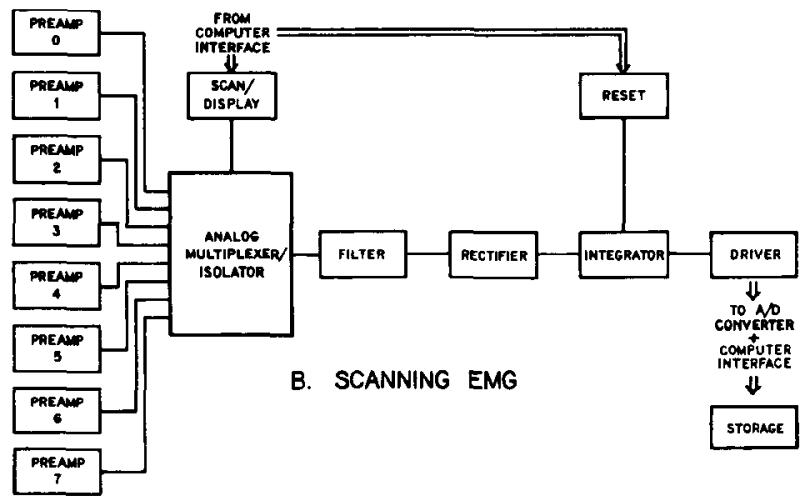

Figure 1. Block diagrams indicating the signal-processing stages for a standard EMG unit and the SEMG instrument. 
measured, the SEMG was constructed for use under direct computer control. A PDP-8/e minicomputer, ${ }^{2}$ along with the State Systems interface ${ }^{3}$ and accompanying software (see Software Control section), directs the scanning process and the analog-to-digital (A/D) conversion.

\section{PRINCIPLES OF OPERATION}

Manufacturers of EMG instruments incorporate a wide variety of bandwidths, integration time constants, feedback modalities, and amplifier designs (Rugh \& Schwitzgebel, 1977). Choice of specific SEMG design parameters will be explained as each functional block is described. Six electronic modules make up the SEMG: the power supplies, the scan/display module, the preamplifier module (up to eight in number), the analog multiplexer/isolator module, the active filter, and the rectifier/ integrator/driver module. An optional module for the delivery of auditory biofeedback is included.

\section{Power Supplies}

Figure 2 shows the schematic diagram for the two SEMG power supplies. To insure that the subject is protected from leakage currents from the line source, a separate battery supply powers all circuitry that interfaces with the participant (the front circuitry).

The computer interface circuitry and signal-processing (rear) circuitry are powered by two supplies derived from the line. Power is furnished to the digital logic (TTL) by the 5-V supply, which incorporates an integrated circuit regulator, the LM309K.

The rear-circuitry analog design utilizes an LM325N dual-polarity tracking regulator to generate $\pm 15 \mathrm{~V}$ at 1 A (see Figure 2). Details on the LM325N may be found in the Voltage Regulator Handbook (National Semiconductor Corporation, 1975).

Two ground symbols are employed. The front circuitry employs a "pseudoground," that is, an indifferent common point of connection. The rear circuitry employs a true earth ground, which should be connected to the ground wire on a grounded plug and to the metal cabinet containing the SEMG. At no point do the pseudoground and earth ground come into contact because of the danger of electric shock. The risk of dangerous shock is negligible, given both the highimpedance circuitry and the optical isolation techniques employed.

The rear-circuitry supplies, excluding switches and transformers, are easily mounted on one $10 \times 15 \mathrm{~cm}$ circuit card. ${ }^{4}$ A small heat sink is employed for the LM309K; a bent piece of sheet metal is sufficient. No heat sinking is required for the $2 \mathrm{~N} 3055$ transistors.

\section{Channel Scan/Display Module}

This circuit, shown in Figure 3, serves three purposes. First, it accepts the 3-bit binary code from the State Systems interface, which selects the EMG channel being sampled; three SPST toggle switches allow manual channel selection for calibration purposes. Second, the module provides control signals for the analog multiplexer/isolator. Third, the circuit supplies a frontpanel light-emitting diode (LED) display, furnished by the SN7445N BCD-to-decimal decoder chip, which indicates the channel that is currently being sampled.

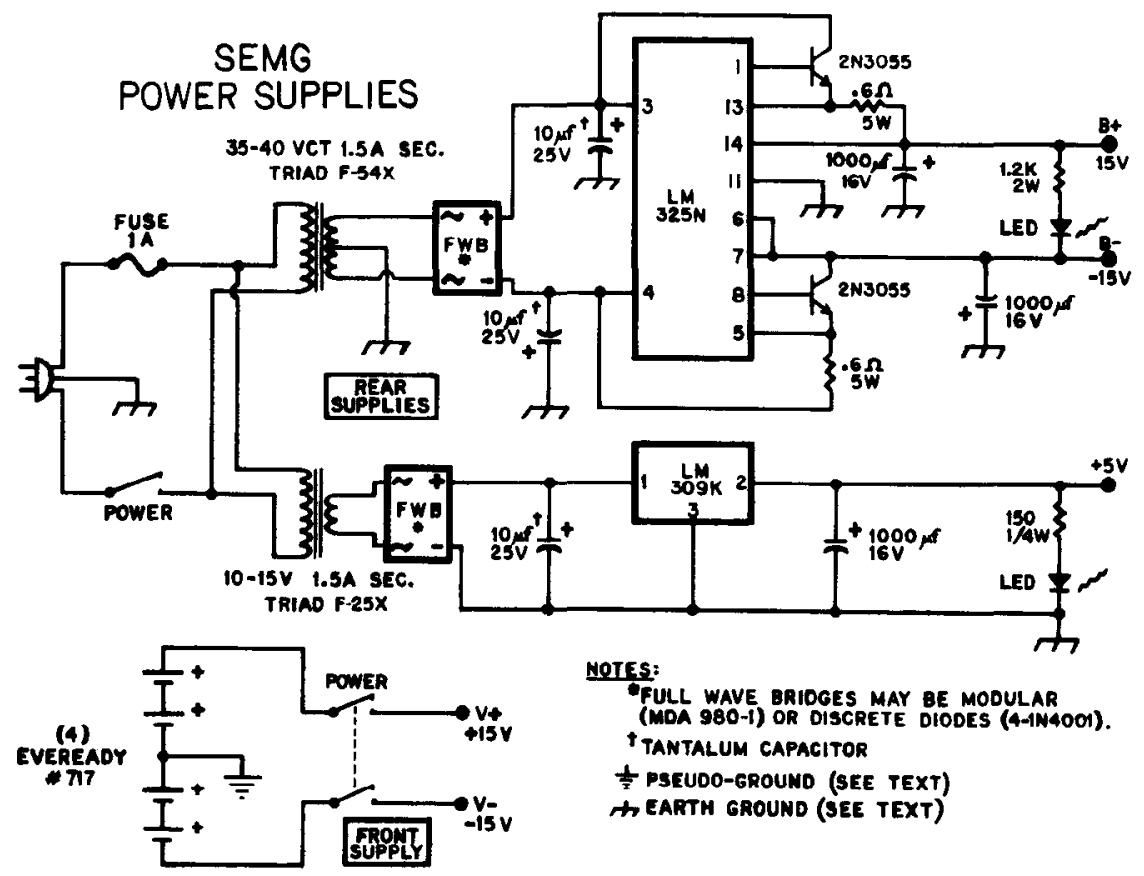

Figure 2. Circuit diagram of the power supplies employed in the SEMG. The use of two separate grounds should be noted. 


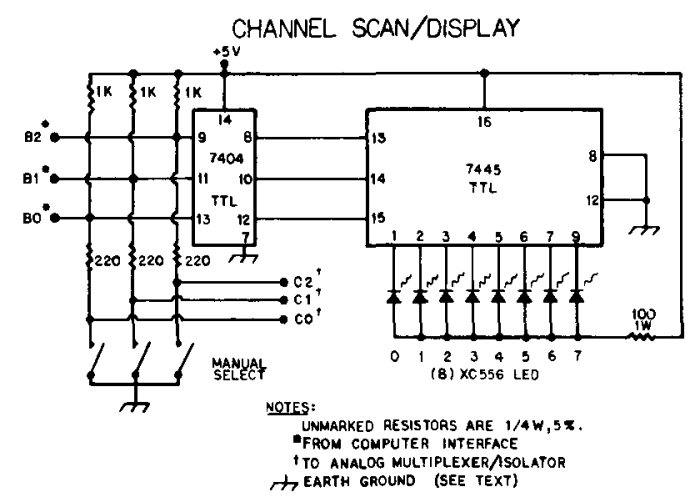

Figure 3. Circuit diagram of the channel scan/display module. Points B0, B1, and B2 are connected to an output word of the State Systems PDP-8 I/O interface.

The module connects directly to the 7406 gates on an output word of the State Systems interface.

\section{SEMG Preamplifier Module}

This module is the most critical portion of any EMG system, and a considerable technical foreword is necessary before detailing the proposed circuit.

Measurement of most biopotentials requires specialized amplifier circuits known as instrumentation amplifiers (Ott, Note 1). Use of instrumentation amplifiers as EMG preamplifiers involves compromises in order to provide generally workable circuits. For example, the capacitors that couple the electrodes to the preamplifier in many designs may be made larger in order to increase the preamplifier's low-frequency response, but only at the expense of increased recovery time from transients (such as the jiggling of an electrode), and degraded common-mode rejection (Strong, 1970). Similarly, the noise output of an instrumentation amplifier is, for practical purposes, proportional to its bandwidth (Soderquist, Note 2). Therefore, in order to minimize noise, the MAP-detection bandwidth should be limited as much as possible without sacrificing important EMG information.

The question of bandwidth selection in electromyography deserves special mention. The frequencies selected for an EMG passband do not correspond to the firing rates of motor units, which rarely exceed $50 \mathrm{~Hz}$ (Basmajian, 1974), but are designed to include frequencies that contain the most energy for the average myopotential. Several studies have undertaken the spectral analysis of MAP waveforms. Hayes (1960) found that reliable measurements of average MAP levels from the biceps brachii can be obtained with a passband from $20 \mathrm{~Hz}$ to 100 or $200 \mathrm{~Hz}$, with frequencies above $200 \mathrm{~Hz}$ contributing little to the total voltage. Kaiser and Petersén (1963), recording from the biceps, show that a $50-$ to $400-\mathrm{Hz}$ passband encompasses most of the myopotential energy. The data may be interpreted to show that even a relatively narrow passband centered near $200 \mathrm{~Hz}$ results in linear measurements of tensional level, although the recorded MAP levels are somewhat attenuated. For averaged MAP recording, wherein changes in tension are being assessed, a narrow passband is probably optimal because its noise-limiting advantages are certain.

Grossman and Weiner (1966) accurately maintain that if peak MAP levels are to be ascertained, a wider bandwidth is advisable. Hayes (1960) found that the 100 - to $1,000-\mathrm{Hz}$ components "determine the wave form of the spike potentials sometimes encountered at low levels of tension" (p. 752). Thus, it seems that research involving measurement of absolute MAP activity or MAP waveform analysis, or research in which measurement linearity (as opposed to mere monotonicity) is critical, should employ instrumentation with extended measurement bandwidth.

The use of electromyography in biofeedback entails special considerations in passband selection. Due to both volume conductance phenomena and the frequencydependent transconductance properties of the skin (see Venables \& Martin, 1967; Waring, 1974), EMG recording from one muscle group is contaminated by activity from contiguous muscles. This fact may be advantageous when treating tension-related disorders with biofeedback. As Basmajian (1976) states, "a wide source of myopotentials is much to be preferred as a reflection of general nervous tension" (p. 370). On the other hand, biofeedback of activity in specific muscles (e.g., in neuromuscular rehabilitation) is aided by the selection of a narrow passband that attenuates the effects of capacitive transconductance. DeBacher and Basmajian (1977) state that specificity of recording site is increased by rolling off the frequencies below $90 \mathrm{~Hz} .^{5}$

The preamplifier presented here has a passband of $30-1,000 \mathrm{~Hz}$ (-3-dB points), in order that the module might be generally adaptable. The SEMG system allows further bandwidth limitation after the preamplifiers (in the active filter and rectifier/integrator/driver modules).

The circuit, shown in Figure 4, uses the National Semiconductor LF352D integrated circuit instrumentation amplifier. ${ }^{6}$ The LF352D employs high input impedance junction field effect transistors (JFET) as the differential input stage to an otherwise bipolar amplifier. Use of this circuit eliminates the need for precise matching of gain resistors formerly required to insure high common-mode rejection in older discrete instrumentation amplifier designs. Complete specifications on the LF352D may be found in the Linear Data Book (National Semiconductor Corporation, 1976a).

The resistor " $T$ " formed by the two 1-megohm and the 10-megohm resistors provides a return path for input bias currents (Figure 4), stabilizing the amplifier and affording a differential input resistance of $2 \times(1 \mathrm{megohm})=2$ megohm, and a common-mode input resistance of 10 megohm $+.5 \times(1$ megohm $)$ $=10.5$ megohm. The high common-mode resistance 


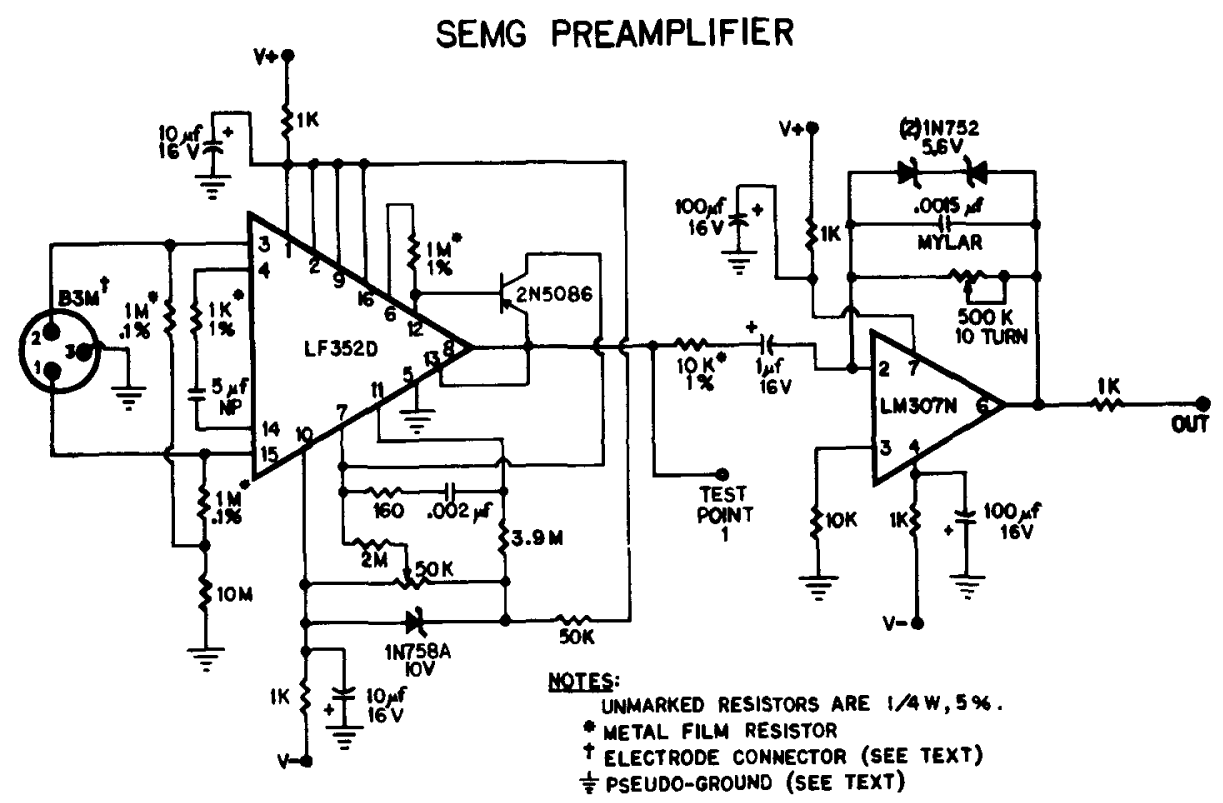

Figure 4. Circuit diagram of the SEMG preamplifier (one of eight maximum), configured for gain of 10,000 and 30 to $1,000-\mathrm{Hz}$ passband.

serves to maximize common-mode rejection (Strong, 1970).

The 1-kohm resistor at Pin 4 sets the gain of the LF352D at 1,000, while the 5-microF nonpolarized capacitor (Cornell-Dubilier Mylar Type WMF05W5 or equivalent ${ }^{7}$ ) that continues to Pin 14 establishes a 6-dB/octave rolloff below $30 \mathrm{~Hz}$ (Ott, Note 1). This arrangement effectively couples the JFET inputs to the electrodes without the use of large blocking capacitors with their associated transient-recovery problems.

The 50-kohm potentiometer need only be a one-turn trimmer that is used to null the input offset voltage (see Calibration section). The 2N5086 PNP transistor functions to suppress large signal transients and to prevent them from damaging the LF352D output stage.

The output of the instrumentation amplifier feeds an inverting-mode, gain-of-10, operational amplifier (the $\mathrm{LM} 307 \mathrm{~N}$ ) via an $\mathrm{R}-\mathrm{C}$ circuit that provides a 6-dB/octave rolloff below $16 \mathrm{~Hz}$. This coupling eliminates multiplication of offset errors from the output of the LF352D, and further attenuates low-frequency noise.

The gain of 10 for the second stage was selected so that a 100-microV EMG signal would cause a $1-\mathrm{V}$ signal at the output of the LM307N. This $1-\mathrm{V}$ reference level is useful for subsequent signal processing. The .0015-microF capacitor in the LM307N feedback loop provides a $6-\mathrm{dB} /$ octave rolloff above $1 \mathrm{kHz}$ only when the stage gain is 10 . The stage gain may be varied by adjusting the 500-kohm potentiometer from 1 (with 1,000 microV producing a $1-\mathrm{V}$ output) to 50 (with 20 microV producing a $1-\mathrm{V}$ output), depending upon the application. However, the feedback capacitor must be changed to restore the $1-\mathrm{kHz}$ rolloff at the new gain. The value may be computed according to Equation 1:

$$
\mathrm{f}_{\mathrm{o}}=1 /(2 \pi \mathrm{RC}),
$$

wherein $f_{o}$ is the corner frequency (at which rolloff begins, in hertz), $\mathrm{R}$ is the potentiometer setting (in ohms) for the desired gain, and $C$ is the value for the capacitor to be obtained (in farads). The $1-\mathrm{kHz}$ rolloff is optional at this stage, and the prescient researcher may wish to install different passbands between or across SEMG channels before active filtering occurs. Equation 1 may also be employed to adjust the lower passband limit by changing the value of the capacitor in the R.C circuit at the input of the LM307N.

Zener diodes in the feedback loop serve to hard limit the output of the LM307N to approximately $6.2 \mathrm{~V}$ (Faulkenberry, 1977). This is necessary because of certain constraints imposed by the analog multiplexer/ isolator module to be considered in the next section. Some limiting occurs with outputs as low as $2.5 \mathrm{~V}$, due to imperfect diode breakdown. This results in waveform distortion and nonlinearity of measurement above $2.5 \mathrm{~V}$. However, since the full-scale reference voltage has been set at $1 \mathrm{~V}$, this distortion is not a problem in practice. A 1 -kohm resistor at the preamplifier output has been included to protect the $\mathrm{LM} 307 \mathrm{~N}$ from possible damage and increased current consumption due to inadvertent shorting of the output to ground.

The entire preamplifier is mounted on a $9 \times 15 \mathrm{~cm}$ circuit card. The eight preamplifiers are mounted in card slots for efficient removal, inspection, and adjustment. Care should be taken when designing the board to separate input and output foils. Also, the 5-microF capacitor is physically large $(1 \times 2 \times 5 \mathrm{~cm})$, and sufficient space should be allowed for it on the circuit card.

The amplifier inputs are wired via twisted or shielded 
cable to B3M shielded audio connectors ${ }^{8}$ mounted on the SEMG front panel, which is itself connected to earth ground. This socket properly mates with the electrode assembly recommended for use in the Electrodes section of this paper. Pin 3 of the B3M connector (Figure 4) is wired to the pseudoground connection on the circuit board for its respective channel.

Total RMS noise voltage (referred to the input) for the preamplifier with $30-$ to $1,000-\mathrm{Hz}$ passband is 6 microV unloaded, and 2.8 microV with a $10-\mathrm{kohm}$ resistor delta connected between the positive and negative inputs, ard from each input to the indifferent point (pseudoground). The shunted-input condition closely resembles that encountered when surface EMG electrodes are in contact with the skin, and this condition has been used in experimental assessment of system noise levels in control procedures (Alexander, 1975). Fortunately, the noise voltage consists largely of high-frequency products, which may be easily reduced in the active filter module.

\section{Analog Multiplexer/Isolator Module}

This circuit uses the control signals supplied by the scan/display module to select the SEMG channel being monitored, and to isolate the analog signals of all channels from any contact with the line-powered rear circuitry. The schematic diagram for the analog multiplexer/isolator module is shown in Figure 5.

The CD4051CN is an eight-channel integrated circuit analog switch that performs the actual selection of the SEMG preamplifier that is to be monitored. Specifica- tions for the CD40510N may be obtained from the National Semiconductor Corporation (1976a). Since the chip functions at a maximum power-supply voltage of $\pm 7.5 \mathrm{~V}$, this power is furnished by two $2: 1$ voltage dividers connected to $\pm \mathrm{V}$. The $\mathrm{CD} 4051 \mathrm{CN}$ may be damaged by input transients greater than the supply voltage; consequently, the preamplifiers are bounded at $\pm 6.2 \mathrm{~V}$.

The full eight-channel capacity of the CD4051CN need not be used. Any number of channels may be installed and the rest added as appropriate.

Channel-select codes are transmitted to the CD4051CN through the MCT2 optoisolators. Signals from the analog switch are linearly coupled to the rear circuitry through the Burr-Brown 3650HG optical isolation amplifier,' herein configured as a unity-gain buffer. This amplifier, as well as affording protection to the research participant from leakage currents, allows the remainder of the MAP signal processing to be accomplished with the line-powered supplies.

\section{Active Filter Module}

Active filtering in the SEMG employs two newly developed National Semiconductor AF100-1CJ active filter circuits. Specifications for the AF100-1CJ may be obtained from the National Semiconductor Corporation (1976b). Figure 6 contains the schematic diagram for the active filter module.

Stage 1 is a $60-\mathrm{Hz}$ notch filter that suppresses stray $60-\mathrm{Hz}$ noise due to imperfect shielding, electrode contact, and common-mode rejection. This filter

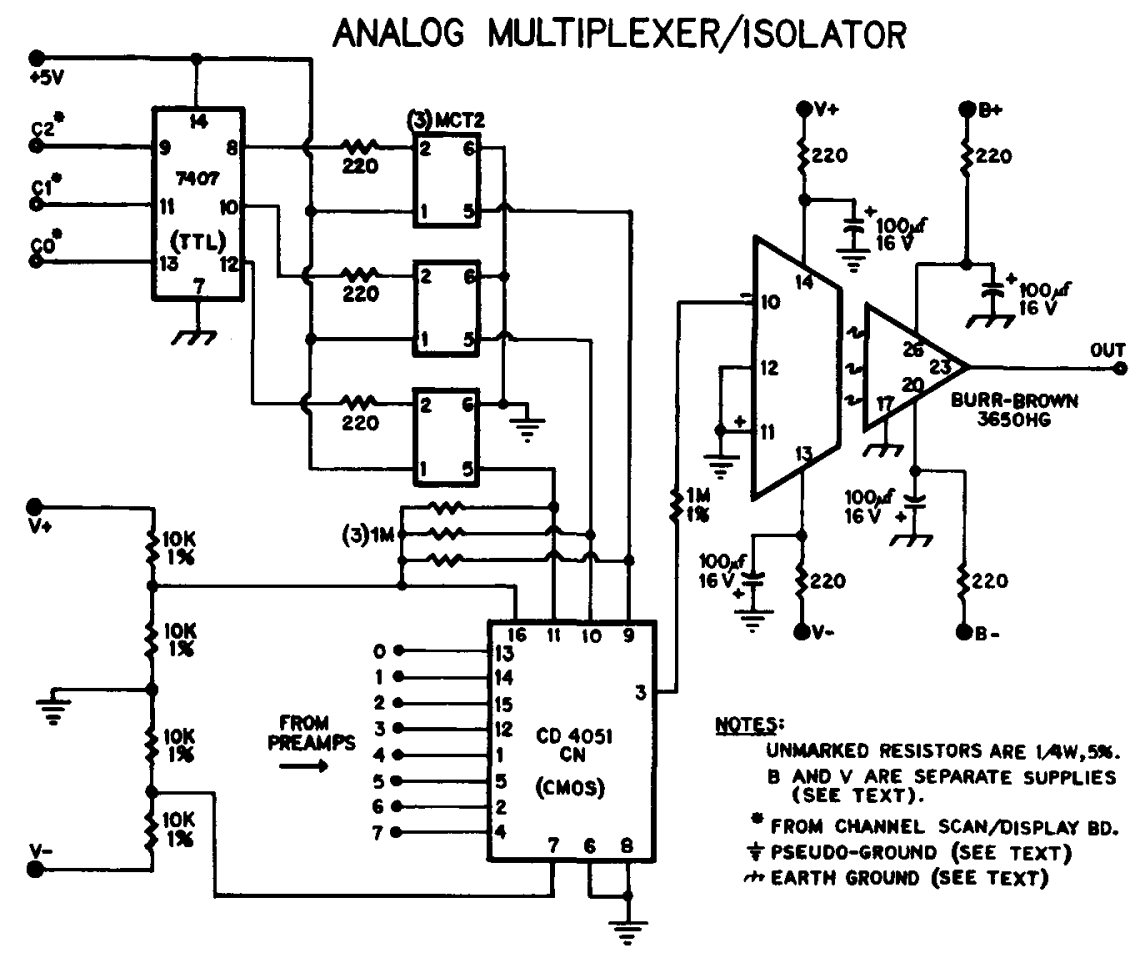

Figure 5. Circuit diagram of the analog multiplexer/isolator module. 


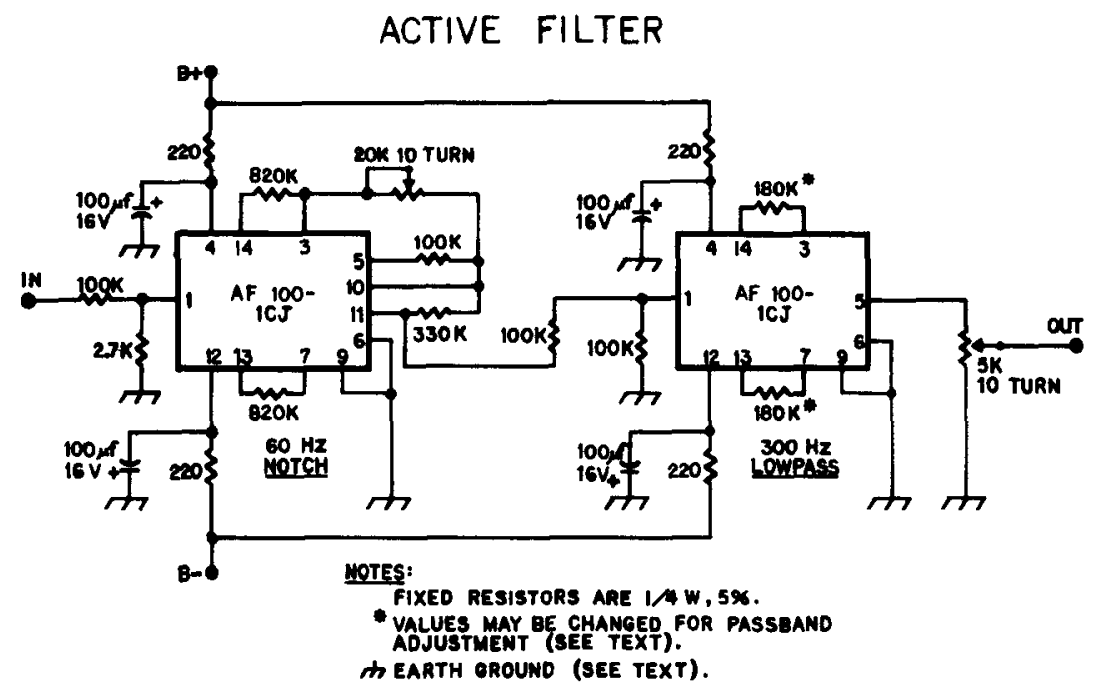

Figure 6. Circuit diagram of the active filter module. This circuit performs $60-\mathrm{Hz}$ rejection and bandpass limiting upon the analog multiplexer/isolator signal, for input to the rectifier/integrator/driver module.

accomplishes $60-\mathrm{Hz}$ attenuation of at least $25 \mathrm{~dB}$, leaving 57- and $63-\mathrm{Hz}$ signals unattenuated. The 20-kohm 10-turn potentiometer is used to null the filter at $60 \mathrm{~Hz}$.

Stage 2 is a Butterworth-type low-pass filter that provides 12-dB/octave attenuation above a selected cutoff frequency. Low-pass filtering introduced at this point will affect all channels of preamplification.

It is here that the previous discussion of EMG bandwidth vs. noise becomes critical. The Stage 2 filter provides a corner frequency of approximately $300 \mathrm{~Hz}$, which reduces total RMS noise voltage to under 1.2 microV on each channel (with 10-kohm delta input configuration). This compromise was judged ideal for our laboratory, in which moderate-level tensional patterning is being studied.

Researchers investigating low-level EMG phenomena may wish to restrict further the upper passband limit to $200 \mathrm{~Hz}$ (the lower limit for this configuration of the AF100-1CJ), and thus reduce total noise (input conditions as above) to under $.8 \mathrm{microV}$. Conversely, any passband upper limit from $300 \mathrm{~Hz}$ to the preamplifiers' imposed cutoffs $(1 \mathrm{kHz}$ for the values shown in the schematic diagram) may be installed.

Modification of the passband upper limit is accomplished by changing the two equal-valued resistors that connect Pins 3 and 14, and Pins 7 and 13, according to the formula in Equation 2:

$$
f_{c}=50.33 \times 10^{6} / R
$$

wherein $f_{c}$ is the corner frequency of interest (in hertz), and $R$ is the value (in ohms) to be used for each resistor in order to attain the desired frequency.

High-pass filtering at this point in the signal processing is accomplished entirely through the capacitive coupling in the preamplifiers, in which rolloffs steeper than $6 \mathrm{~dB} /$ octave offer little advantage in terms of noise reduction. An additional opportunity to change the high-pass corner frequency to above $30 \mathrm{~Hz}$ is available in the rectifier/integrator/driver module.

The active filter module mounts compactly on one $7 \times 15 \mathrm{~cm}$ circuit board. Instructions on adjustment of the $60-\mathrm{Hz}$ notch filter are given in the Calibration section.

\section{Rectifier/Integrator/Driver Module}

This circuit serves three functions: transformation of the bipolar MAP signals from the active filter module to a varying level suitable for integration, integration of the rectified MAP signals over a specified time interval, and buffering of the integrated signal for input to an A/D converter. The schematic diagram for this module is presented in Figure 7.

Linear rectification of signals with amplitudes less than $1 \mathrm{~V}$ does not permit the use of standard diode bridge configurations, since silicon diodes have a forward-bias voltage drop (conduction threshold) of $.6 \mathrm{~V}$. This drawback necessitates the use of a configuration called a precision rectifier, or absolute value circuit, in which the diodes are inserted in the feedback loop of an operational amplifier so that the voltage drop is reduced by the high amplifier gain. The circuit shown is a variation of that described by Graeme (Note 3). It employs two LM307N operational amplifiers that act as a voltage follower and an inverter, respectively, for alternating signal polarities. The amplifier outputs are paralleled to provide negative-going full-wave rectification of the incoming MAP signals (the negative rectification is necessary to achieve a positive integrated voltage from the inverting-mode integrator stage). The precision rectifier accurately processes signal levels from the low- 


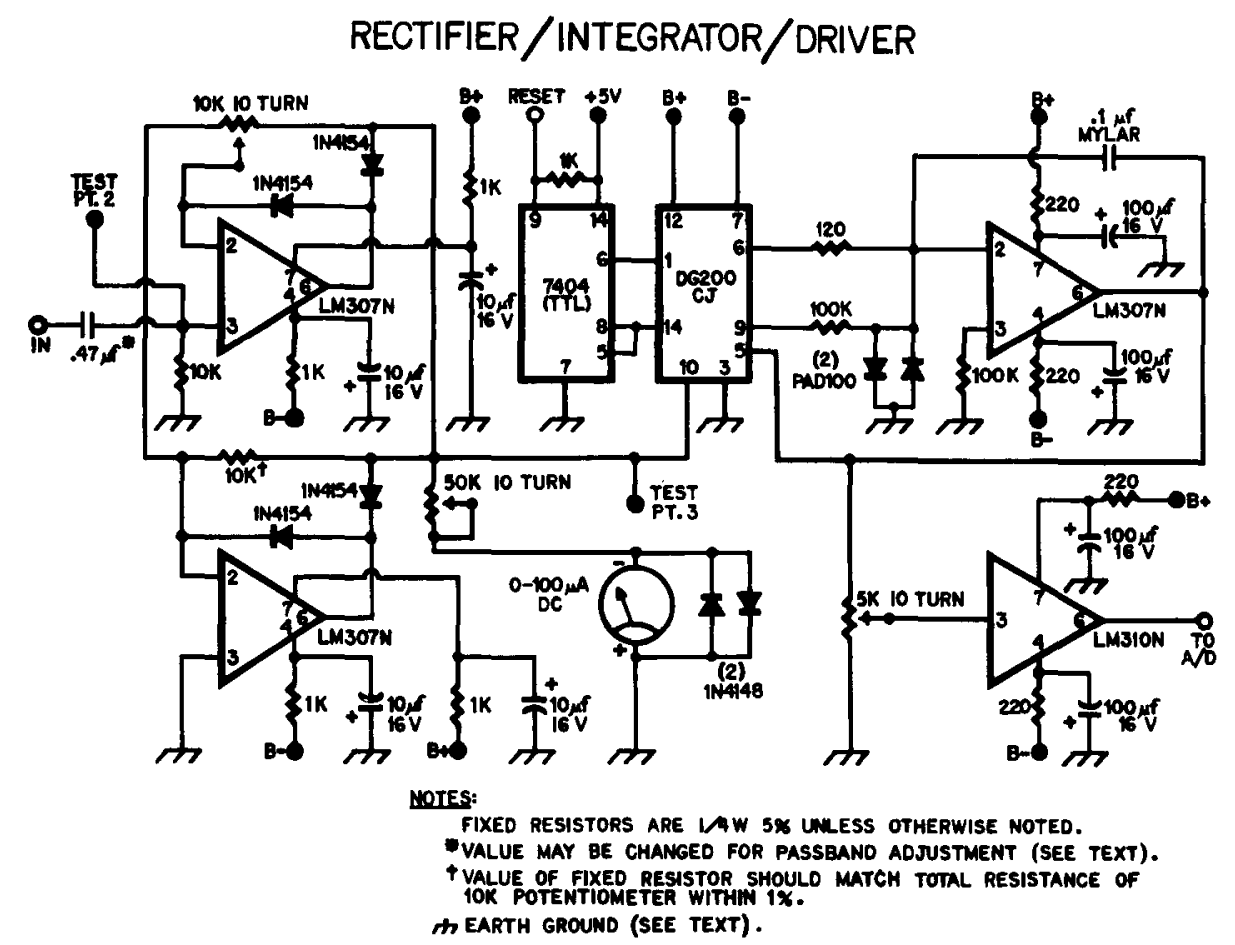

Figure 7. Circuit diagram of the rectifier/integrator/driver module. Input to this section is from the active filter module. The "RESET" input is connected to an output word of the State Systems PDP-8 I/O interface (see Software Control section in text).

millivolt range to approximately $10 \mathrm{~V}$. The gain of the circuit is variable from one to several thousand, depending upon the adjustment of the $10-\mathrm{kohm} 10$ turn potentiometer. Directions for gain adjustment of the precision rectifier are furnished in the Calibration section.

The input to the precision rectifier is an R-C circuit designed to neutralize offset voltages from the isolation amplifier and active filter circuits, and to provide a 6-dB/octave high-pass cutoff at about $30 \mathrm{~Hz}$. The value of the input capacitor may be changed according to Equation 1, if further modification of the passband lower limit is desired.

The output of the precision rectifier drives both the integrator section and a calibration meter. The 50-kohm potentiometer allows accurate adjustment of the meter, following adjustment of the gain of the precision rectifier. The two $1 \mathrm{~N} 4148$ diodes act to protect the meter movement from damaging voltage transients.

The SEMG design requires the use of an integrator that samples a channel's output for a fixed time interval (under computer control), then resets to zero before another channel is scanned (refer to Figure 1). The circuit that accomplishes this function is appropriately termed a resetting integrator; the particular circuit employed here is a variation of one proposed by Shaeffer (Note 4). Two sections of an SN7404N hex inverter toggle a Siliconix DG200CJ dual-SPST analog switch. ${ }^{10}$ In the "integrate" mode (RESET input pulled to $+5 \mathrm{~V}$ by the $1-\mathrm{kohm}$ resistor), one switch within the DG200CJ carries the signal from the precision rectifier to the $\mathrm{LM} 307 \mathrm{~N}$ integrator. Upon receipt of a reset pulse (RESET input shunted to ground by the 7406 output of the State Systems interface), the alternate switch is enabled and the positive integrated voltage is shunted to ground through the $120-\mathrm{ohm}$ resistor. Signals are integrated through the $.1-\mathrm{microF}$ Mylar capacitor. The two Siliconix PAD100 (picoampere diodes) limit the integrated voltage to $+15 \mathrm{~V}$, thus preventing damage to the DG200CJ.

The output of the integrator connects via the 5-kohm front-panel mounted potentiometer to an LM310N voltage follower (unity-gain buffer). The potentiometer allows precise scaling of the integrated voltage (in microvolt-seconds), which is delivered via the LM310N to the A/D converter being employed. The LM310N buffers the output of the potentiometer, providing a low-impedance output that allows the use of a long cable for connecting the SEMG output to the A/D converter. The LM310N output should be coupled to the A/D converter through shielded cable and appropriate connectors.

\section{CALIBRATION}

Calibration of the SEMG requires the use of a stable sine-wave generator capable of covering a range from 10 to $1,000 \mathrm{~Hz}$ (e.g., the Hewlett-Packard $241 \mathrm{~A}^{11}$ ), a digital frequency counter (e.g., the Hewlett-Packard 
5321B) to measure precisely the generator output frequency, and a volt-ohmmeter with at least $1 \%$ accuracy (e.g., the Data Precision Model $175^{12}$ ).

The preamplifiers are calibrated first. For this purpose, a microvolt-level input signal is best achieved through a voltage-divider circuit as follows: Using a Switchcraft A3F connector plug (which mates with the B3M input sockets), Pins 2 and 3 are wired to the plug ground connection. A $100-0 h m 1 \%$ resistor proceeds from Pin 1 to the plug ground. A 1-megohm 1\% resistor goes from Pin 1 to the inner conductor of a shielded cable connected at the other end to the "hot" side of the generator input. The cable shield connects the plug ground to the generator ground. This circuit forms a $10,000: 1$ divider that furnishes a differential signal to the preamplifiers from the generator.

A $200-\mathrm{Hz}$ (or center of chosen passband), 100 microV sine wave $(1.00 \mathrm{~V}$ at the generator output; all voltages in this section refer to "average" or RMS measurement) is fed to each preamplifier to be calibrated. The front-circuitry power supply is enabled. For each preamplifier, the voltage at Test Point 1 (Figure 4) is measured, and the 50-kohm trimmer potentiometer adjusted for a null point $(0 \pm .5 \mathrm{~V})$. Measuring from the preamplifier output, the 500-kohm potentiometer is adjusted for a $1.00 \mathrm{~V}$ output with a preamplifier gain of 10,000 .

After making preliminary checks for obvious short circuits and so on, the rear-circuitry supplies are enabled also. Voltage checks are performed to ascertain the correct operation of all supplies.

Correct operation of the scan/display and analog multiplexer/isolator modules should be verified. The manual channel-select switches are used to select (in binary form) SEMG Channels 0.7. As the 100-microV sine wave from the signal generator is fed to the corresponding preamplifier, $1.00 \mathrm{~V}$ should appear at the input of the active filter module. Crosstalk from unselected channels should be negligible.

Adjustment of the active filter module proceeds. Channel " 0 " is selected, and the 100-microV $200-\mathrm{Hz}$ signal is coupled to its inputs. The 5-kohm potentiometer at the active filter module's output is adjusted for $1.00 \mathrm{~V}$ at Test Point 2 on the rectifier/integrator/ driver board. This adjustment sets the module gain at unity. The signal generator frequency is changed to $60 \mathrm{~Hz}( \pm .1 \mathrm{~Hz}$, as indicated on the frequency counter). The 20-kohm potentiometer on the active filter board is then adjusted for a minimum reading (ACV) at this frequency, as measured from Test Point 2.

Gain adjustment of the precision rectifier is as follows: With Channel " 0 " selected, and with the $100-$ microV $200-\mathrm{Hz}$ signal at its input, a voltage of $1.00 \mathrm{~V}$ is verified at Test Point 2 . Then the 10 -kohm potentiometer is adjusted for $1.00-\mathrm{V}$ reading at Test Point 3 (output of the precision rectifier). The 50-kohm meter-adjustment potentiometer is adjusted for a full- scale reading on the 0 to 100 -microA calibration meter.

Further adjustment of the SEMG integrator/driver circuitry requires knowledge of the desired scan rate and of the input range of the A/D system being employed, since the voltage integral for a channel sample is expressed in Equation 3:

$$
V_{\text {out }}=\frac{-1}{10^{5} C} \int_{t_{1}}^{t_{2}} V_{\text {in }} d t
$$

wherein $\mathrm{V}_{\text {out }}$ is the integrated output voltage, $\mathrm{C}$ is the value of the integration capacitor in farads, $V_{\text {in }}$ is the (negative) output of the precision rectifier in volts, and the $t_{1}-t_{2}$ interval is the measurement duration for one channel in seconds. High scan rates $>20$ channels/sec) may require incrementing the precision rectifier gain (with meter readjustment), in order to achieve usable voltage integrals. Conversely, low scan rates $(<5$ channels/sec) may require a larger value for the integration capacitor. Within ranges of adjustment of the precision rectifier, the $5-\mathrm{kohm}$ potentiometer can be used to scale the integrator output for a wide range of scan rates and $A / D$ converter input requirements. With a 0 - to $5-\mathrm{V}$ A/D converter input range, rates from 5 to 20 channels/sec may be accomplished with the precision rectifier gain at unity, an integration capacitance of $.1 \mathrm{microF}$, and appropriate scaling of the 5-kohm potentiometer.

The offset characteristics of the LM307N used for the integrator are somewhat critical for the accurate measurement of signals close to system noise levels. Low-level measurement nonlinearity may thus be due to an LM307N with out-of-spec offset characteristics, or to offset voltage produced in the precision rectifier circuit. This nonlinearity may be corrected by substituting another LM307N integrator until linearity is obtained.

\section{SCAN RATE AND VARIABILITY}

The EMG signal is an aggregate of discrete yet overlapping MAP. Consequently, as the number of samples of EMG activity per unit time is increased, the variability of the readings increases proportionally. While this variability is cancelled in typical EMG designs through the use of capacitive damping circuits (Tursky, 1964), the SEMG records great fluctuations in EMG activity at higher scan rates. Slow scan rates produce readings that mimic those obtainable with a standard EMG, although with a concomitant loss of simultaneity in the tensional profiles thus obtained.

The rapid fluctuations in the EMG activity within a muscle group may be valuable data in studies of muscle patterning in limb movement. Scan rates up to 50 channels/sec have been successfully used in our laboratory for this purpose. However, studies of tensional patterning under stress or relaxation may 
Table 1

SEMG Specifications

\begin{tabular}{lr} 
Input Resistance Each Channel & \\
Differential (shunted by 3 picoF) & 2 megohm \\
Common-Mode (shunted by 6 picoF) & 10.5 megohm \\
Common-Mode Rejection & $85 \mathrm{~dB}$ \\
Channel Noise (10-kohm input $\triangle$ ) & 1.2 microV \\
60-Hz Rejection & $25 \mathrm{~dB}$ \\
Channel Cro:stalk & $-40 \mathrm{~dB}$ \\
Passband (-3 dB) & $80-300 \mathrm{~Hz}$ \\
\hline
\end{tabular}

permit the use of much slower scan rates; we have employed rates as slow as 1 channel/sec for this purpose.

The loss of simultaneity associated with slow scan rates may be recovered through a process best termed interlaced scanning (cf. the method employed in television raster generation). Through software modification, high scan rates are employed, and several samples for each channel are added before the result is recorded as a datum.

\section{ELECTRODES AND SYSTEM GROUNDING}

Electrodes recommended for use with the SEMG are surface-type silver/silver-chloride disks. Complete assemblies including three electrodes, cable, and $\mathrm{A} 3 \mathrm{~F}$ connector are available from Autogenic Systems, Inc. ${ }^{13}$ For use with the SEMG, the electrode assembly must be modified as follows: The wire from the ground connection on the $\mathrm{A} 3 \mathrm{~F}$ connector is detached and added to Pin 3 (pseudoground).

The SEMG chassis is connected through a three-wire plug to the line ground. The subject is grounded through a metal wrist or ankle strap to the SEMG enclosure. (Refer to the discussion of grounding in biomedical equipment by Strong, 1970.)

\section{SPECIFICATIONS AND COMPONENTRY}

Critical SEMG specifications are supplied in Table 1. Specifications refer in each instance to the "worst" case, such that with careful construction and calibration techniques, and given some variance in component specifications, the listed specifications can be readily obtained. The bandpass curve for the SEMG, with component values as shown in the schematic diagrams, is presented in Figure 8.

A parts list of active componentry for the SEMG is contained in Table 2. All components are available "off the shelf" from the manufacturers' distributors or from retail outlets. ${ }^{14}$

\section{BIOFEEDBACK APPLICATION}

Complex-criterion multiple-muscle biofeedback is readily accomplished with the SEMG. An output word of the State Systems interface drives a digital-to-analog
(D/A) converter that supplies voltage to a feedback module. A test for achievement of the criterion is conducted by the SEMG control program. Upon completion of an entire scan, the program updates the voltage level at the D/A converter output.

The feedback module in Figure 9 supplies a square wave whose frequency is proportional to the input voltage. The circuit directly connects to the D/A converter output, and it is used to provide click or tone biofeedback. The feedback module utilizes a Burr-Brown VFC32KP monolithic voltage-to-frequency converter, buffered by an LM310N voltage follower configured to drive either high-impedance headphones or an audio amplifier. The $5-\mathrm{kohm}$ frequency-adjustment potentiometer allows the full-scale input $(10 \mathrm{~V})$ to produce any frequency from 1 to $5,000 \mathrm{~Hz}$. Input rescaling for $\mathrm{D} / \mathrm{A}$ converter output ranges other than $0.10 \mathrm{~V}$ is accomplished by changing the $39-\mathrm{kohm}$ input resistor to a value that supplies $.25-\mathrm{mA}$ current to VFC32KP Pin 1 at the new full-scale voltage.

\section{SOFTWARE CONTROL}

Although details of software depend upon the computer hardware available, the general features of an adequate program for control of the SEMG can be specified. $^{15}$ The program must determine (1) the duration of the integrator-reset pulse, (2) the length of the dwell time for a selected channel, (3) the number and sequence of channels to be included on each scan (from one to eight), and (4) the number of entire scans desired. Specification of the reset pulse duration through software allows the user to choose the optimal duration for a given integration period. Generally, reset pulse

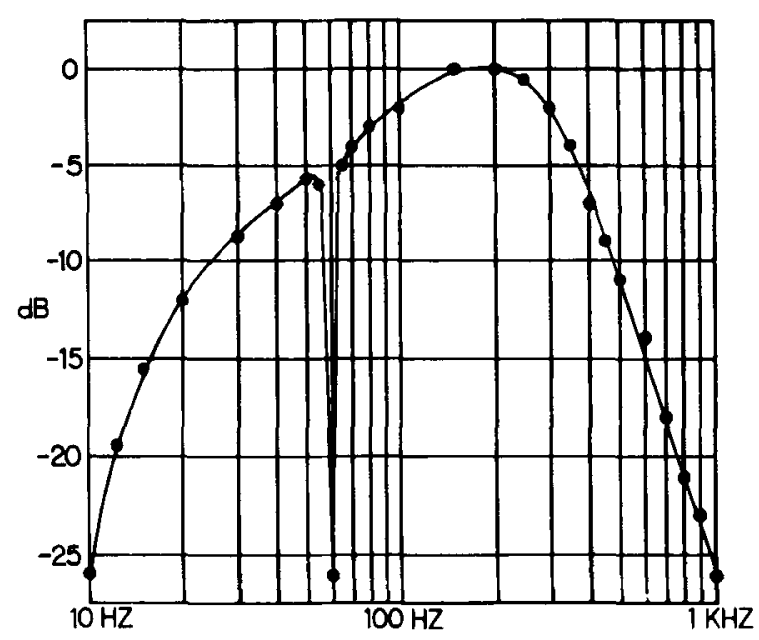

Figure 8. System bandpass curve for the SEMG with proposed circuit values. Reference level $(0 \mathrm{~dB})$ is the voltage produced at Test Point 3 on the rectifier/integrator/driver board by a $100-$ microV $200-\mathrm{Hz}$ sine wave applied to the preamplifier inputs (see Calibration section in text). System response was averaged across preamplifier channels. 
Table 2

Parts List of Active Components for Scanning EMG Modules

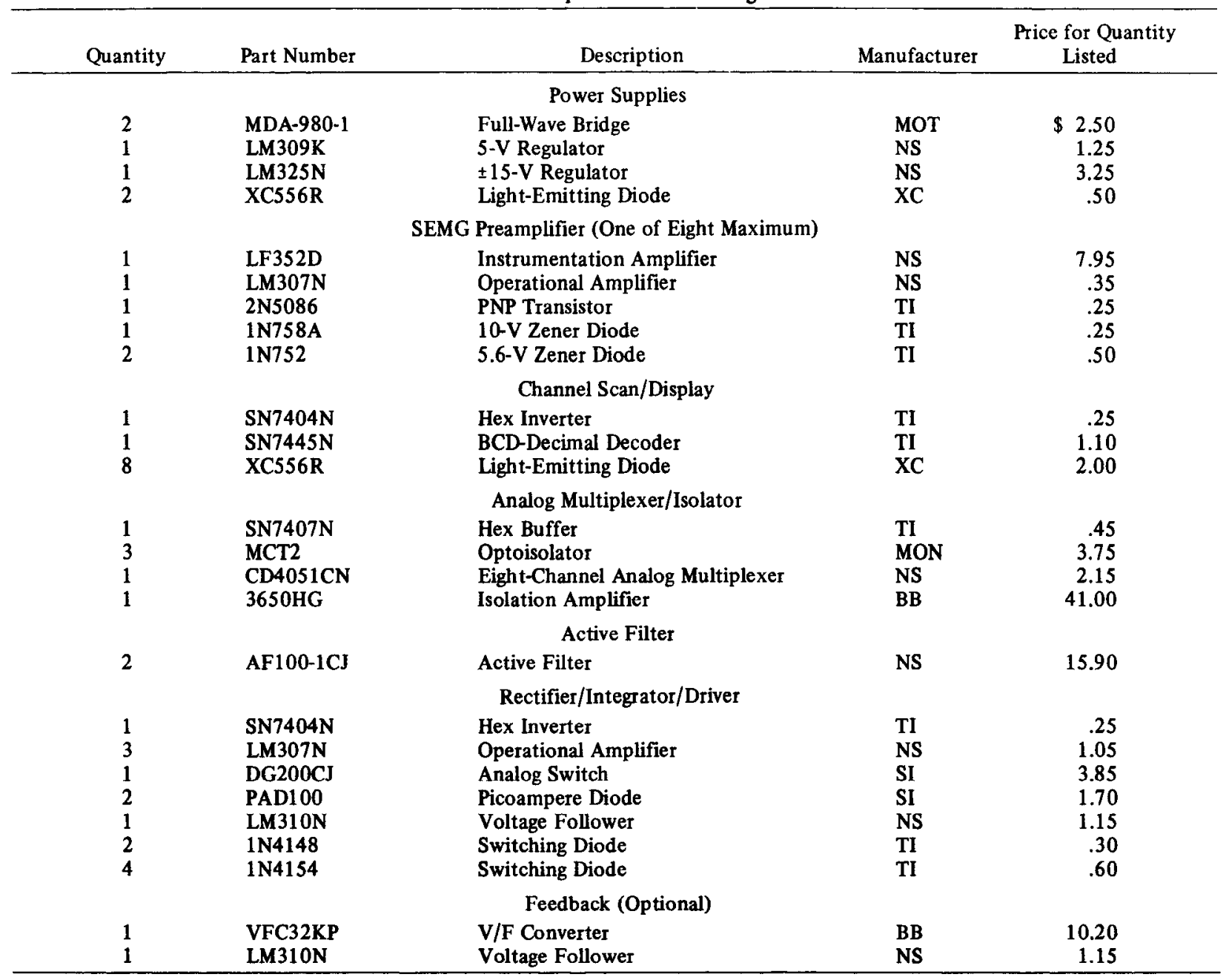

Note-Abbreviations of manufacturers: BB-Burr-Brown, MON-Monsanto, MOT-Motorola, NS-National Semiconductor, SISiliconix, TI-Texas Instruments, XC-Xciton.

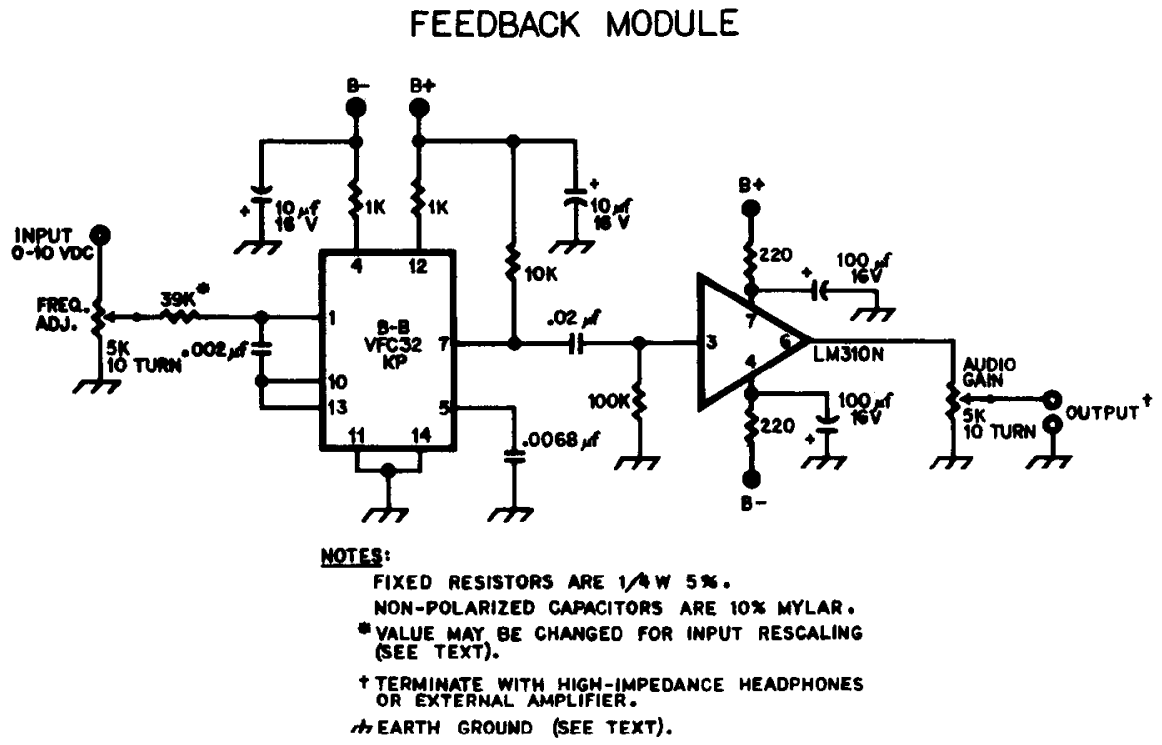

Figure 9. Circuit diagram of the optional feedback module, configured for 10-V full-scale input voltage. 
durations of $.01-.02 \mathrm{sec}$ are adequate to reset the integrator completely.

After completion of the reset pulse, the program selects a specific channel and times the integration period. Thus, scan rate is effectively determined by the sum of the reset pulse duration and channel integration period. A/D conversion of the integrated EMG signal for a selected channel commences at the end of the integration. For details on the analog signal processing, see Fowler and Leberer (1977). Routine software manipulations then store the digitized measurement. Implementation of complex-criterion multiple-muscle biofeedback requires an additional algorithm for combining measurements from designated muscle groups.

\section{REFERENCE NOTES}

1. Ott, W. E. Instrumentation amplifiers: Versatile differential input gain blocks. Application Note AN-75. Tucson, Ariz: Burr-Brown Research Corporation, 1974.

2. Soderquist, D. Minimization of noise in operational amplifier applications. Application Note AN-15. In Linear and conversion I.C. products. Santa Clara, Calif: Precision Monolithics, Inc., 1976.

3. Graeme, J. G. A simplified precision rectifier with variable gain. Application Note AN-78. Tucson, Ariz: Burr-Brown Research Corporation, 1975.

4. Shaeffer, L. CMOS analog switches-A powerful design tool. Application Note AN75-1. In Analog switches and their applications. Santa Clara, Calif: Siliconix, Inc., 1976.

\section{REFERENCES}

Alexander, A. B. An experimental test of assumptions relating to the use of electromyographic biofeedback as a general relaxation technique. Psychophysiology, 1975, 12, 656-662.

Balshan, I. D. Muscle tension and personality in women. Archives of General Psychiatry, 1962, 7, 436-448.

Basmajian, J. V. Muscles alive (3rd ed.). Baltimore, Md: Williams \& Wilkins, 1974.

Basmajian, J. V. Facts vs. myths in EMG biofeedback. Biofeedback and Self-Regulation, 1976, 1, 369-371.

DeBacher, G., \& Basmajian, J. V. EMG feedback strategies in rehabilitation of neuromuscular disorders. In J. Beatty \& H. Legewie (Eds.), Biofeedback and behavior. New York: Plenum, 1977.

FAULKENBERRY, L. M. An introduction to operational amplifiers. New York: Wiley, 1977.

Floyd, W. F., \& Silver, P. H. S. The function of the erectores spinae muscles in certain movements and postures in man. Journal of Physiology, 1955, 129, 184-203.

Fowler, S. C., \& Leberer, M. R. Hardware techniques for analog processing using the State Systems PDP-8 I/O interface. Behavior Research Methods \& Instrumentation, 1977, 9. 210-214.

Grossman, W. I., \& Weiner, H. Some factors affecting the reliability of surface electromyography. Psychosomatic Medicine, 1966, 28, 78-83.

HAYES, K. J. Wave analyses of tissue noise and muscle action potentials. Journal of Applied Physiology, 1960, 15, 749-752.

Kaiser, E., \& Petersén, I. Frequency analysis of muscle action potentials during tetanic contraction. Electromyography, $1963,3,5-17$.

Milner, M., Basmajian, J. V., \& Quanbury, A. O. Multifactorial analyses of walking by electromyography and com- puter. American Journal of Physical Medicine, 1971, 50, 235-258.

National Semiconductor Corporation. Voltage regulator handbook. Santa Clara, Calif: Author. 1975.

National Semiconductor Corporation. Linear data book. Santa Clara, Calif: Author, 1976. (a)

National Semiconductor Corporation. Special function data book. Santa Clara, Calif: Author, 1976. (b)

Rugh, J. D., \& SchwtTzgeBEL, R. L. Variability in commercial electromyographic biofeedback devices. Behavior Research Methods \& Instrumentation, 1977, 9, 281-285.

Schwartz, G. E., Fair, P. L., Salt, P., Mandel, M. R., \& Klerman, G. L. Facial expression and imagery in depression: An electromyographic study. Psychosomatic Medicine, 1976, 38, 337-347.

Strong, P. Biophysical measurements. Beaverton, Oreg: Tektronix, 1970.

TURSKY, B. Integrators as measuring devices of bioelectric output. Clinical Pharmacology and Therapeutics, 1964, 5, 887-892.

Venables, P. H., \& Martin, I. A manual of psychophysiological methods. New York: American Elsevier, 1967.

W ARING, W. Observing signals from nerve and muscle. In H. A. Miller \& D. C. Harrison (Eds.), Biomedical electrode technology. New York: Academic Press, 1974.

\section{NOTES}

1. The block diagrams are somewhat stylized. In EMG designs that employ battery power supplies, isolation may occur after signal processing for interfacing with external equipment (e.g., strip-chart recorders), or it may be omitted altogether Some EMG instruments may omit a discrete integration stage, instead employing either a damped meter movement or a simple R-C integrating network (Tursky, 1964) to lend stability to the readout.

2. PDP is a registered trademark of the Digital Equipment Corporation, Maynard Massachusetts 01754.

3. State Systems, Inc., P.O. Box 2215, Kalamazoo, Michigan 49001.

4. A set of negative transparencies for all SEMG circuitry for use with negatively photosensitized printed circuit material may be obtained at cost ( $\$ 9.50)$ from the first author.

5 . In the present SEMG design, the $90-\mathrm{Hz}$ cutoff is best instituted in each preamplifier by changing the 1-microF capacitor at LM307N Pin 2 to a value of .15 microF.

6. National Semiconductor Corporation, 2900 Semiconductor Drive, Santa Clara, California 95051.

7. Cornell-Dubilier Electronics Division, Federal Pacific Electric Company, 150 Avenue L, Newark, New Jersey 07101.

8. Switchcraft, Inc., 5527 North Elston Avenue, Chicago, Illinois 60630 .

9. Burr-Brown Research Corporation, International Airport Industrial Park, Tucson, Arizona 85374.

10. Siliconix, Inc., 2201 Laurelwood Road, Santa Clara, California 95054.

11. Hewlett-Packard, 1507 Page Mill Road, Palo Alto, California 94304.

12. Data Precision Corporation, Audubon Road, Wakefield, Massachusetts 01880.

13. Type M-1 EMG electrode set is available at $\$ 30$ each from Autogenic Systems, Inc., 809 Allston Way, Berkeley, California 94710 . Type $\mathbf{M - 2}$ adhesive contact disks for electrode attachment are available at $\$ 16.50$ for 297 disks (minimum order).

14. James Electronics, 1021 Howard Avenue, San Carlos, California 94070.

15. An assembly list for the SEMG control program is available from the first author. 